International Journal of Pure and Applied Mathematics

Volume 86 No. 2 2013, 403-410

ISSN: 1311-8080 (printed version); ISSN: 1314-3395 (on-line version)

url: http://www.ijpam.eu

doi: http://dx.doi.org/10.12732/ijpam.v86i2.14

ijpam.eu

\title{
APPROXIMATION OF GENERALIZED BINOMIAL BY POISSON DISTRIBUTION FUNCTION
}

\author{
K. Teerapabolarn \\ Department of Mathematics \\ Faculty of Science \\ Burapha University \\ Chonburi, 20131, THAILAND
}

\begin{abstract}
The Stein-Chen method and the $w$-function associated with generalized binomial random variable are used to determine a non-uniform bound on Poisson approximation to generalized binomial distribution function. Some numerical examples are given to illustrate applications of the result.
\end{abstract}

AMS Subject Classification: 62E17, 60F05

Key Words: generalized binomial distribution function, Poisson approximation, Stein-Chen method

\section{Introduction}

The generalized binomial distribution in this study was introduced by Dwass [3]. It is a generalized discrete distribution that covers binomial, hypergeometric and Pólya distributions. It depends on four parameters, $A, B, n$ and $\alpha$, where $A$ and $B$ are positive, $n$ is a positive integer, $\alpha$ is an arbitrary real number satisfying $(n-1) \alpha \leq A+B$, and $A^{(i)}$ and $B^{(i)}$ are not negative for $i=1, \ldots, n$, where $x^{(i)}=x(x-\alpha) \cdots(x-(i-1) \alpha)$. Let $X$ be the generalized binomial random variable. Then, following Dwass [3], its probability function is of the

Received: March 9, 2013

(c) 2013 Academic Publications, Ltd. url: www.acadpubl.eu 
form

$$
\begin{aligned}
& p_{X}(x)=\left(\begin{array}{l}
n \\
x
\end{array}\right) \frac{A^{(x)} B^{(n-x)}}{(A+B)^{(n)}}, x=0, \ldots, n . \\
&=\left(\begin{array}{l}
n \\
x
\end{array}\right) \frac{[A(A-\alpha) \cdots(A-(x-1) \alpha)][B(B-\alpha) \cdots(B-(n-x-1) \alpha)]}{(A+B)(A+B-\alpha) \cdots(A+B-(n-1) \alpha)}, \\
& x=0, \ldots, n .
\end{aligned}
$$

The mean and variance of $X$ are $\mu=\frac{n A}{A+B}$ and $\sigma^{2}=\frac{n A B(A+B-n \alpha)}{(A+B)^{2}(A+B-\alpha)}$, respectively. It is known that both binomial and hypergeometric distributions can be approximated by Poisson distribution under certain conditions on their parameters. Thus, if the conditions on the parameters of generalized binomial and the Poisson distributions are satisfied, then the generalized binomial distribution can also be approximated by the Poisson distribution. For example, Wongkasem et al. [7] gave a uniform bound on Poisson approximation to generalized binomial distribution as follows:

$$
\left|\sum_{x \in \Omega} p_{X}(x)-\sum_{x \in \Omega} \wp_{\lambda}(x)\right| \leq\left(1-e^{-\lambda}\right) \frac{B(n-1) \alpha+A(A+B-\alpha)}{(A+B)(A+B-\alpha)},
$$

where $\Omega \subseteq\{0, \ldots, n\}, \wp_{\lambda}(x)=\frac{e^{-\lambda} \lambda^{\mathrm{x}}}{x !}, \lambda=\frac{n A}{A+B}$ and $A \geq(n-1)(-\alpha)$ when $\alpha<0$. Teerapabolarn [5] gave a non-uniform bound on this pointwise approximation, that is,

$$
\left|p_{X}(0)-e^{-\lambda}\right| \leq \lambda^{-1}\left(\lambda+e^{-\lambda}-1\right) \frac{B(n-1) \alpha+A(A+B-\alpha)}{(A+B)(A+B-\alpha)}
$$

and for $x_{0} \in\{1, \ldots, n\}$,

$$
\left|p_{X}\left(x_{0}\right)-\wp_{\lambda}\left(x_{0}\right)\right| \leq \min \left\{1-e^{-\lambda}, \frac{\lambda}{x_{0}}\right\} \frac{B(n-1) \alpha+A(A+B-\alpha)}{(A+B)(A+B-\alpha)} .
$$

Let us consider (1.3), for $x_{0} \in\{0, \ldots, n\}$ and $\Omega=\left\{0, \ldots, x_{0}\right\}$, then we have

$$
\left|\mathbb{F}_{X}\left(x_{0}\right)-\mathbb{P}_{\lambda}\left(x_{0}\right)\right| \leq\left(1-e^{-\lambda}\right) \frac{B(n-1) \alpha+A(A+B-\alpha)}{(A+B)(A+B-\alpha)},
$$

where $\mathbb{F}_{X}\left(x_{0}\right)$ and $\mathbb{P}_{\lambda}\left(x_{0}\right)$ are the generalized binomial and Poisson distribution functions at $x_{0}$. It is observed that the bound in (1.6) does not depend on $x_{0}$, or it is a uniform bound with respect to $x_{0}$. So, the uniform bound in (1.6) may not be sufficient for measuring the accuracy of the approximation. In this case, a non-uniform bound with respect to $x_{0}$ is more appropriate. 
In this paper, we use the Stein-Chen method and $w$-function associated with the generalized binomial random variable to give a non-uniform bound on Poisson approximation to the generalized binomial distribution function, which are mentioned in Sections 2 and 3, respectively. In Section 4, some numerical examples are given to illustrate applications of the result, and the conclusion are presented in the last section.

\section{Method}

Stein [4] introduced a powerful method for bounding the error in the normal approximation. This method was first developed and applied to the Poisson approximation by Chen [2], which is refer to as the Stein-Chen method. Following [6], Stein's identity for Poisson approximation to the generalized binomial distribution function is of the form

$$
\mathbb{F}\left(x_{0}\right)-\mathbb{P}_{\lambda}\left(x_{0}\right)=\mathbb{E}\left[\lambda f_{x_{0}}(X+1)-X f_{x_{0}}(X)\right],
$$

where $x_{0} \in \mathbb{N} \cup\{0\}, X$ is the generalized binomial random variable and $f_{x_{0}}$ is defined as in [6]. Teerapabolarn [6] showed that $\left|\Delta f_{x_{0}}(x)\right|=\mid f_{x_{0}}(x+1)-$ $f_{x_{0}}(x) \mid \leq \min \left\{\lambda^{-1}\left(1-e^{-\lambda}\right), \frac{1}{x_{0}}\right\}$ for $x>0$.

For $w$-function associated with the generalized binomial random variable, Wongkasem et al. [7] gave the $w$-function as the following lemma.

Lemma 2.1. Let $w(X)$ be $w$-function associated with the generalized binomial random variable $X$ and $p_{X}(x)>0$ for every $0 \leq x \leq n$. Then

$$
w(x)=\frac{(n-x)(A-x \alpha)}{(A+B) \sigma^{2}} \geq 0, x=0, \ldots, n,
$$

where $\sigma^{2}=\frac{n A B(A+B-n \alpha)}{(A+B)^{2}(A+B-\alpha)}$.

The following relation is an important property for proving the result, which was stated by Cacoullos and Papathanasiou [1]. If a non-negative integer-valued random variable $X$ have $p_{X}(x)>0$ for every $x$ in the support of $X$ and have finite variance $0<\sigma^{2}<\infty$, then

$$
\mathbb{E}[(X-\mu) f(X)]=\sigma^{2} \mathbb{E}[w(X) \Delta f(X)],
$$

for any function $f: \mathbb{N} \cup\{0\} \rightarrow \mathbb{R}$ for which $\mathbb{E}|w(X) \Delta f(X)|<\infty$, where $\Delta f(x)=f(x+1)-f(x)$. For $f(x)=x$, we have that $\mathbb{E}[w(X)]=1$. 


\section{Result}

The following theorem presents a non-uniform bound for approximation of the generalized binomial distribution function by the Poisson distribution function.

Theorem 3.1. Let $\lambda=\frac{n A}{A+B}>0$ and $A \geq(n-1)(-\alpha)$ for $\alpha<0$. Then we have the following:

$$
\left|\mathbb{F}_{X}(0)-e^{-\lambda}\right| \leq \lambda^{-1}\left(\lambda+e^{-\lambda}-1\right) \frac{B(n-1) \alpha+A(A+B-\alpha)}{(A+B)(A+B-\alpha)}
$$

and for $x_{0} \in\{1, \ldots, n\}$,

$$
\left|\mathbb{F}_{X}\left(x_{0}\right)-\mathbb{P}_{\lambda}\left(x_{0}\right)\right| \leq \min \left\{1-e^{-\lambda}, \frac{\lambda}{x_{0}}\right\} \frac{B(n-1) \alpha+A(A+B-\alpha)}{(A+B)(A+B-\alpha)}
$$

Proof. From (2.1), it follows that

$$
\mathbb{F}_{X}\left(x_{0}\right)-\mathbb{P}_{\lambda}\left(x_{0}\right)=\lambda \mathbb{E}\left[\Delta f_{x_{0}}(X)\right]-\mathbb{E}\left[(X-\mu) f_{x_{0}}(X)\right],
$$

Since $\mathbb{E}[w(X)]=1$ and $\mathbb{E}\left|w(X) \Delta f_{x_{0}}(X)\right|=\mathbb{E}\left[w(X)\left|\Delta f_{x_{0}}(X)\right|\right]<\infty$. Thus, by $(2.3)$, we have

$$
\begin{aligned}
\left|\mathbb{F}_{X}\left(x_{0}\right)-\mathbb{P}_{\lambda}\left(x_{0}\right)\right| & \leq\left|\lambda \mathbb{E}\left[\Delta f_{x_{0}}(X)\right]-\sigma^{2} \mathbb{E}\left[w(X) \Delta f_{x_{0}}(X)\right]\right| \\
& \leq \mathbb{E}\left\{\left|\lambda-\sigma^{2} w(X)\right|\left|\Delta f_{x_{0}}(X)\right|\right\} \\
& \leq \sup _{x \geq 1}\left|\Delta f_{x_{0}}(x)\right| \mathbb{E}\left|\lambda-\sigma^{2} w(X)\right|
\end{aligned}
$$

Because

$$
\begin{aligned}
\lambda-\sigma^{2} w(x) & =\frac{n A}{A+B}-\frac{(n-x)(A-x \alpha)}{A+B} \\
& =\frac{[(n-x) \alpha+A] x}{A+B} \\
& \geq 0, \\
E\left|\lambda-\sigma^{2} w(X)\right| & =\lambda-\sigma^{2} E[w(X)] \\
& =\lambda-\sigma^{2} \\
& =\lambda \frac{B(n-1) \alpha+A(A+B-\alpha)}{(A+B)(A+B-\alpha)} .
\end{aligned}
$$


Therefore

$$
\left|\mathbb{F}_{X}\left(x_{0}\right)-\mathbb{P}_{\lambda}\left(x_{0}\right)\right| \leq \min \left\{1-e^{-\lambda}, \frac{\lambda}{x_{0}}\right\} \frac{B(n-1) \alpha+A(A+B-\alpha)}{(A+B)(A+B-\alpha)} .
$$

Hence, the result is obtained.

Remark. Because $\lambda^{-1}\left(\lambda+e^{-\lambda}-1\right)<1-e^{-\lambda}$ and $\min \left\{1-e^{-\lambda}, \frac{\lambda}{x_{0}}\right\} \leq$ $1-e^{-\lambda}$. It can be seen that the bound in Theorem 3.1 is sharper than the bound in (1.6).

\section{Numerical Examples}

The following numerical examples are given to illustrate how well Poisson distribution function approximates generalized binomial distribution function.

4.1. Suppose that $n=10, A=30, A+B=1000$ and $\alpha=0$, then $\lambda=0.30$. Numerical results in (1.6) and Theorem 3.1 are as follows:

(i). For uniform bound,

$$
\left|\mathbb{F}_{X}\left(x_{0}\right)-\mathbb{P}_{\lambda}\left(x_{0}\right)\right| \leq 0.007775453, x_{0}=0, \ldots, 10
$$

(ii). For non-uniform bound,

$$
\left|\mathbb{F}_{X}\left(x_{0}\right)-\mathbb{P}_{\lambda}\left(x_{0}\right)\right| \leq \begin{cases}0.004081822 & \text { if } x_{0}=0 \\ 0.007775453 & \text { if } x_{0}=1,2,3 \\ \frac{0.03}{x_{0}} & \text { if } x_{0}=4, \ldots, 10\end{cases}
$$

4.2. Suppose that $n=30, A=50, A+B=1000$ and $\alpha=0$, then $\lambda=1.470588235$. Numerical results in (1.6) and Theorem 3.1 are as follows:

(i). For uniform bound,

$$
\left|\mathbb{F}_{X}\left(x_{0}\right)-\mathbb{P}_{\lambda}\left(x_{0}\right)\right| \leq 0.037755379, x_{0}=0, \ldots, 30
$$

(ii). For non-uniform bound,

$$
\left|\mathbb{F}_{X}\left(x_{0}\right)-\mathbb{P}_{\lambda}\left(x_{0}\right)\right| \leq \begin{cases}0.023345950 & \text { if } x_{0}=0 \\ 0.037755379 & \text { if } x_{0}=1 \\ \frac{0.049019608}{x_{0}} & \text { if } x_{0}=2, \ldots, 30\end{cases}
$$


4.3. Suppose that $n=10, A=30, A+B=1000$ and $\alpha=1$, then $\lambda=0.30$. Numerical results in (1.6) and Theorem 3.1 are as follows:

(i) For uniform bound,

$$
\left|\mathbb{F}_{X}\left(x_{0}\right)-\mathbb{P}_{\lambda}\left(x_{0}\right)\right| \leq 0.010040375, x_{0}=0, \ldots, 10
$$

(ii) For non-uniform bound,

$$
\left|\mathbb{F}_{X}\left(x_{0}\right)-\mathbb{P}_{\lambda}\left(x_{0}\right)\right| \leq \begin{cases}0.005270821 & \text { if } x_{0}=0 \\ 0.010040375 & \text { if } x_{0}=1,2,3 \\ \frac{0.038738739}{x_{0}} & \text { if } x_{0}=4, \ldots, 10\end{cases}
$$

4.4. Suppose that $n=30, A=50, A+B=1000$ and $\alpha=1$, then $\lambda=1.470588235$. Numerical results in (1.6) and Theorem 3.1 are as follows:

(i). For uniform bound,

$$
\left|\mathbb{F}_{X}\left(x_{0}\right)-\mathbb{P}_{\lambda}\left(x_{0}\right)\right| \leq 0.058600497, x_{0}=0, \ldots, 30 .
$$

(ii). For non-uniform bound,

$$
\left|\mathbb{F}_{X}\left(x_{0}\right)-\mathbb{P}_{\lambda}\left(x_{0}\right)\right| \leq \begin{cases}0.036235481 & \text { if } x_{0}=0 \\ 0.058600497 & \text { if } x_{0}=1 \\ \frac{0.076083819}{x_{0}} & \text { if } x_{0}=2, \ldots, 30\end{cases}
$$

4.5. Suppose that $n=10, A=30, A+B=1000$ and $\alpha=-1$, then $\lambda=0.30$. Numerical results in (1.6) and Theorem 3.1 are as follows:

(i) For uniform bound,

$$
\left|\mathbb{F}_{X}\left(x_{0}\right)-\mathbb{P}_{\lambda}\left(x_{0}\right)\right| \leq 0.005515057, x_{0}=0, \ldots, 10 .
$$

(ii) For non-uniform bound,

$$
\left|\mathbb{F}_{X}\left(x_{0}\right)-\mathbb{P}_{\lambda}\left(x_{0}\right)\right| \leq \begin{cases}0.002895198 & \text { if } x_{0}=0 \\ 0.005515057 & \text { if } x_{0}=1,2,3 \\ \frac{0.021278721}{x_{0}} & \text { if } x_{0}=4, \ldots, 10\end{cases}
$$


4.6. Suppose that $n=30, A=50, A+B=1000$ and $\alpha=-1$, then $\lambda=1.470588235$. Numerical results in (1.6) and Theorem 3.1 are as follows:

(i). For uniform bound,

$$
\left|\mathbb{F}_{X}\left(x_{0}\right)-\mathbb{P}_{\lambda}\left(x_{0}\right)\right| \leq 0.016951093, x_{0}=0, \ldots, 30 .
$$

(ii). For non-uniform bound,

$$
\left|\mathbb{F}_{X}\left(x_{0}\right)-\mathbb{P}_{\lambda}\left(x_{0}\right)\right| \leq \begin{cases}0.010481669 & \text { if } x_{0}=0 \\ 0.016951093 & \text { if } x_{0}=1 \\ \frac{0.022008412}{x_{0}} & \text { if } x_{0}=2, \ldots, 30 .\end{cases}
$$

In view of numerical examples 4.1-4.6, it is found that the results (in case of non-uniform bound) are better than the results (in case of uniform bound).

\section{Conclusion}

The non-uniform bound in Theorem 3.1 is a criteria for measuring the accuracy of the approximation, that is, if it is small, then a good Poisson approximation for the generalized binomial distribution function is obtained. The bound in this study is sharper than those reported in [7].

\section{References}

[1] T. Cacoullos, V. Papathanasiou, Characterization of distributions by variance bounds, Statist. Probab. Lett., 7 (1989), 351-356.

[2] L.H.Y. Chen, Poisson approximation for dependent trials, Ann. Probab., 3 (1975), 534-545.

[3] M. Dwass, A generalized binomial distribution, Amer. Statistician, 33 (1979), 86-87.

[4] C.M. Stein, A bound for the error in normal approximation to the distribution of a sum of dependent random variables, Proc. Sixth Berkeley Sympos. Math. Statist. Probab., 3 (1972), 583-602.

[5] K. Teerapabolarn, A pointwise approximation of generalized binomial by Poisson distribution, Appl. Math. Sciences, 6 (2012), 1095-1104. 
[6] K. Teerapabolarn, A non-uniform bound on Poisson approximation by $w$ functions, Int. J. Pure Appl. Math., 78 (2012), 1063-1075.

[7] P. Wongkasem, K. Teerapabolarn, R. Gulasirima, On Approximating a generalized binomial by binomial and Poisson distributions, Int. J. Statist. Systems, 3 (2008), 113-124. 